\title{
A Novel Three-Component System in Visible Light Photo-induced Polymerization
}

\author{
Makoto Kume, Yasushi Ohe and Takao Taguchi \\ Materials Research Laboratory, Technical Research Institute, Toppan Printing Co., Ltd. \\ 4-2-3 Takanodaiminami, Sugito-machi, Kitakatsushika-gun, Saitama-ken 345-8508, Japan \\ Makoto.Kume@toppan.co.jp
}

Keywords: visible light, photopolymer, ketocoumarin, onium salt, borate salt, photo-DSC

\section{Introduction}

Several photopolymers which are insolubilized or solubilized by irradiation with visible light, have been attractive for applications to photo-imaging , three-dimensional stereo-lithography, holography, dental materials and so on.[1]-[3] Most well known sensitized photoinitiator systems are based on photo-induced electron-transfer reactions and generate free radicals by visible light irradiation. [4],[5]

Our previous paper dealt with a diaryliodonium salt / 3-ketocoumarin system, which is based on photo-induced electron-transfer reaction and generates effectively active free radicals and active cations in a photopolymer which has applications in holography.[6] However, the photopolymer layer containing this photoinitiator system after curing was colored, it has a yellowish aspect caused by the color of the sensitizing dye. It is a serious problem in a field demanded colorless and transparent usage materials.

In this paper, an addition of borate salt known as electron-donating initiator [7],[8] to this system was attempt to improve the color aspect of the photopolymer after curing without a lowering of photosensitivity.

\section{Experimental}

The photopolymer system is prepared as follows. To a $46 \mathrm{wt} \%$ solution of bisphenol A type epoxy resin in 2-butanone was added a 50-100 wt\% radical polymerizable monomer based on the epoxy resin, to which were added 4,4'-bis- (tertbutylphenyl)iodonium hexafluorophosphate (BBI,
$1.0-7.5 \mathrm{wt} \%$ to the epoxy resin), 3,3'-carbonylbis (7-diethylaminocoumarin) (KCD, $0.1-0.25 \mathrm{wt} \%$ to the epoxy resin) and tetrabutylammonium triphenylbutylborate (BTPB, $0-1.0 \mathrm{wt} \%$ to the epoxy resin).

Photopolymerization studies were conducted with a photo-DSC (Du Pont, 830 Differential Photocalorimeter). To obtain the heat of photopolymerization $(\Delta \mathrm{H})$, photosensitive solution was placed in an aluminum pan and dried at $80^{\circ} \mathrm{C}$ for $10 \mathrm{~min}$. The sample was irradiated with visible light from a $200 \mathrm{~W}$ high pressure mercury lamp passed through a $\mathrm{L}-42$ cut filter. Irradiation time was 1 or $2 \mathrm{~min}$.

To evaluate the photosensitivity of the polymer system, a sample for testing was prepared as follows. The photopolymer solution was coated on an anode-oxidized aluminum plate or a glass plate with doctor blade (gap: 3 mil), and dried at $80^{\circ} \mathrm{C}$ for $10 \mathrm{~min}$. The coating thickness after drying was about $12 \mu \mathrm{m}$. There after, a $10 \mathrm{wt} \%$ aqueous solution of poly(vinyl alcohol) was spin-coated on the photosensitive layer. The plate was exposed to an $\mathrm{Ar}^{+}$laser beam (Spectra Physics, model-2020) operating at 488-nm. After the laser exposed, the plate was heated at $80^{\circ} \mathrm{C}$ for $10 \mathrm{~min}$ (post-exposure baking; PEB). In addition, it was developed with acetone after removing the PVA layer, or it was cured with UV-light at $2 \mathrm{~J} / \mathrm{cm}^{2}$. Photosensitivity was evaluated to the desired exposure energy to make a polymerized spot on the aluminum plate having a diameter which was the same as that of laser beam after developing.

YI value (JIS/K7103) of photopolymerized layer 
on the glass plate was measured using Color Measuring System (NIPPON DENSYOKU, SZ590) after UV-curing.

Spectral sensitivity curves were obtained by use of Grating Spectrograph (NALUMI MR-23-I) with $\mathrm{Xe}$ arc light source and was irradiated for $5 \mathrm{~min}$.

\section{Results and Discussion}

Figures 1 and 2 show the experimental results which were obtained by photo-DSC analyses and photosensitivity measurement of KCD / BBI / BTPB system. It was noticed that the $\Delta H$ and the sensitivity in this system were more effective than in a KCD / BBI system by addition of a small amount of borate salt.

However, comparing the $\triangle \mathrm{H}$ of KCD / BBI system with that of KCD / BTPB system, there were almost similar results between the two photoinitiator systems as shown in Figure 3.

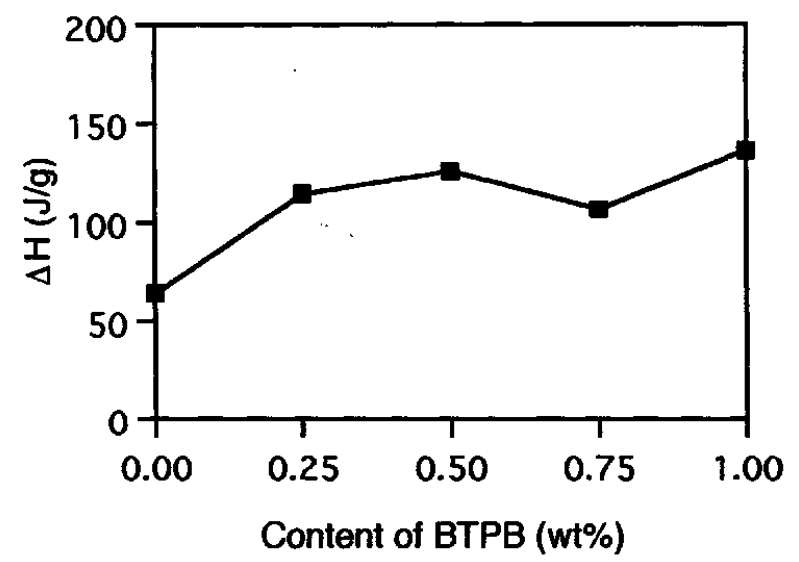

Figure 1. $\Delta \mathrm{H}$ of acrylic monomer determined by photoDSC in epoxy resin initiated with $7.5 \mathrm{wt} \%$-BBI / 0.1 wt\%-KCD system including various contents of BTPB.

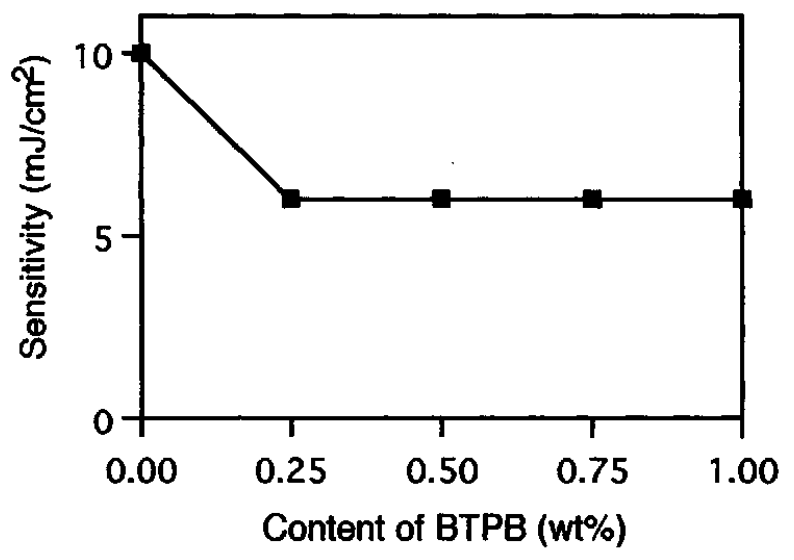

Figure 2. Sensitivity of photopolymer consisted of epoxy resin / acrylic monomer initiated with $7.5 \mathrm{wt} \%$ $\mathrm{BBI} / 0.1 \mathrm{wt} \%-\mathrm{KCD}$ system including various contents of BTPB using a laser light at 488-nm.
If there is no interaction among the $\mathrm{KCD} / \mathrm{BBI}$ / BTPB system, the sensitivity of the photopolymer containing $7.5 \mathrm{wt} \%$-BBI and $0.5 \mathrm{wt} \%$-BTPB should be near to that of $8.0 \mathrm{wt} \%$-BBI. Since actual photosensitivity in BBI / BTPB system was about twice higher than of $10 \mathrm{wt} \%-\mathrm{BBI}$ system as shown in Figure 4, it is expected that there are some interactions in the three-component system.

On the other hand, the YI value of the film made from $0.1 \mathrm{wt} \%-\mathrm{KCD}$ system was decreased, compared with 0.25 wt\%-KCD system on the photopolymerized layer containing BBI and BTPB as shown in the Table. It's confirmed that the photopolymerized layer becomes almost colorless and transparent. However, the sensitivity of the photopolymer from $0.1 \mathrm{wt} \%-\mathrm{KCD}$ containing 0.5 wt\%-BTPB system was higher than of $0.25 \mathrm{wt} \%$ KCD system.

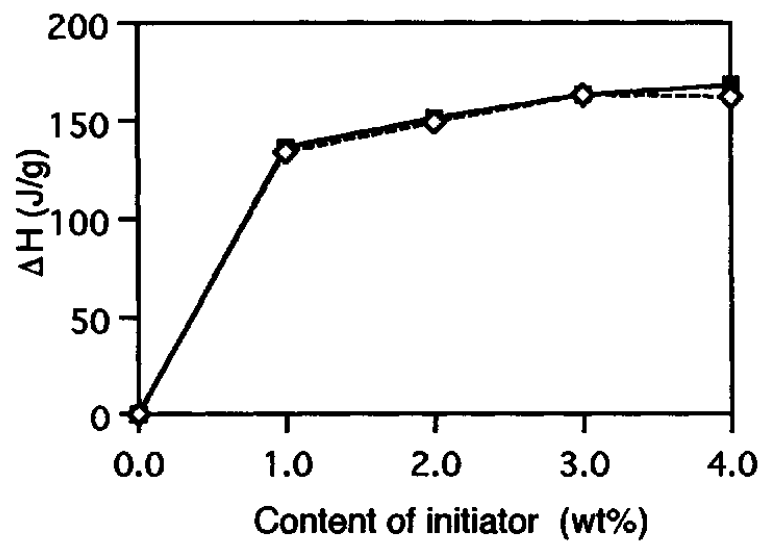

Figure 3. $\Delta \mathrm{H}$ of acrylic monomer sensitized with 0.1 wt\%-KCD system including various contents of initiator. $\longrightarrow$; BBI, - - >--; BTPB.

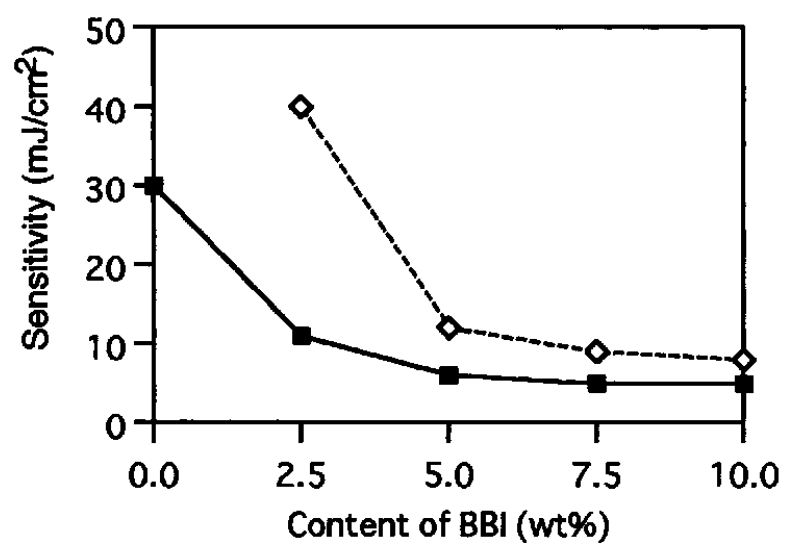

Figure 4. Sensitivity of photopolymer consisted of epoxy resin /acrylic monomer/0.1 wt\%-KCD initiated with various contents of BBI system: _and including $0.5 \mathrm{wt} \%$-BTPBsystem:-- --- 
Table: Sensitivity and Yellowness Index (YI) of Photopolymer Consisted of Epoxy Resin / Acrylic Monomer System

\begin{tabular}{ccccc}
\hline \multicolumn{2}{c}{ Content of photoinitiator system } & Sensitivity $\left(\mathrm{mJ} / \mathrm{cm}^{2}\right)$ & Yellowness Index (YI) \\
\hline $0.25 w t \%-K C D$ & $7.5 w t \%-B B I$ & $0.0 w t \%-B T P B$ & 6 & 2.65 \\
& $5.0 w t \%-B B I$ & $0.0 w t \%-B T P B$ & 7 & 3.88 \\
\hline $0.1 w t \%-K C D$ & $7.5 w t \%-B B I$ & $0.0 w t \%-B T P B$ & 10 & 1.72 \\
& & $0.5 w t \%-B T P B$ & 5 & 2.05 \\
& $5.0 w t \%-B B I$ & $0.0 w t \%-B T P B$ & 12 & 2.08 \\
& & $0.5 w t \%-B T P B$ & 6 & 2.27 \\
\hline
\end{tabular}

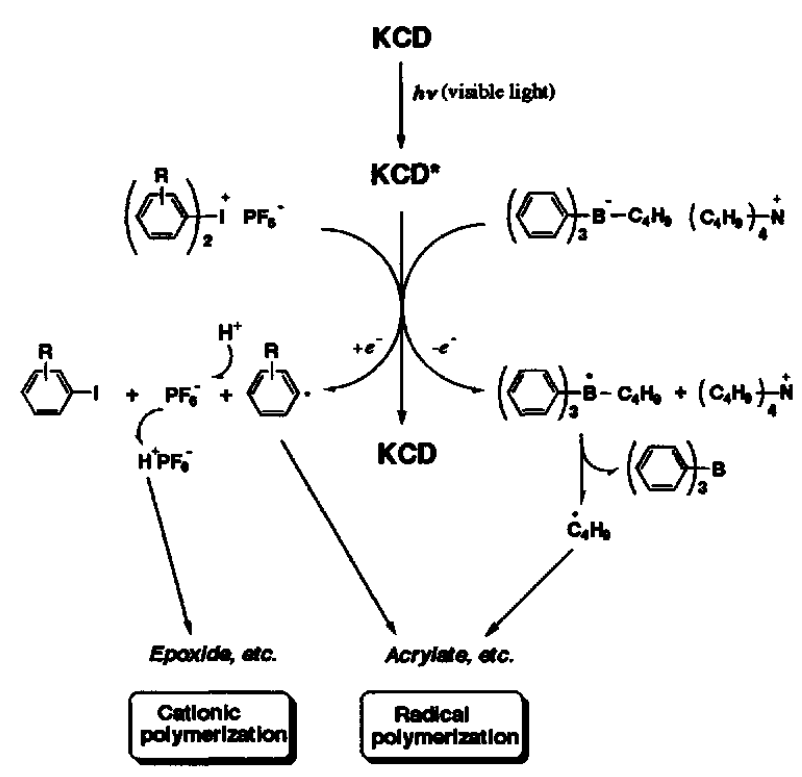

Scheme 1. Reaction pathway for KCD and BBI system added BTPB.

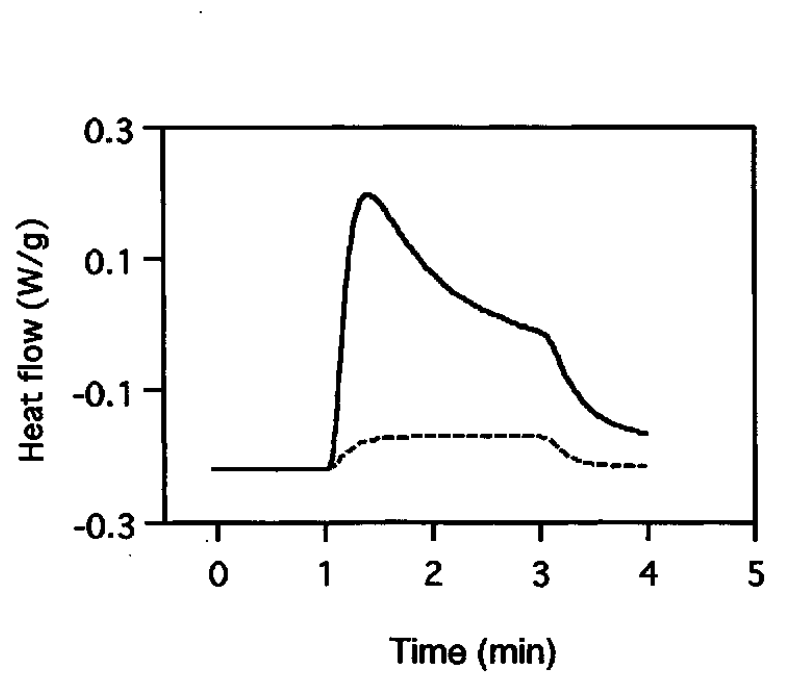

Figure 5. Photo-DSC curves for mixture of epoxy monomer and photoinitiation system. BBI/KCD system,--- - ; BTPB/KCD system.

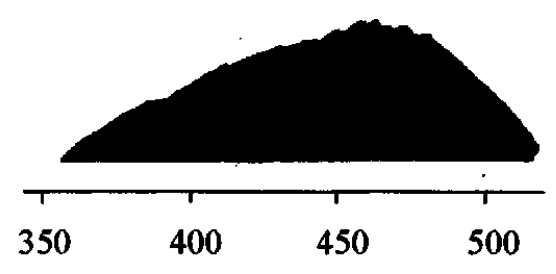

(A)

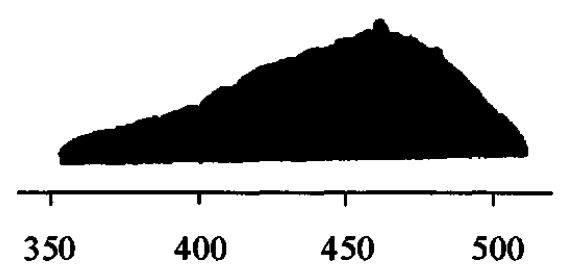

(B)

Figure 6. Spectral sensitivity curves of photopolymers containing 7.5wt\%-BBI and $0.25 w t \%-K C D(A)$ and $7.5 w t \%-B B I$, $0.5 w t \%-B T P B$ and $0.1 w t \%-K C D(B)$.

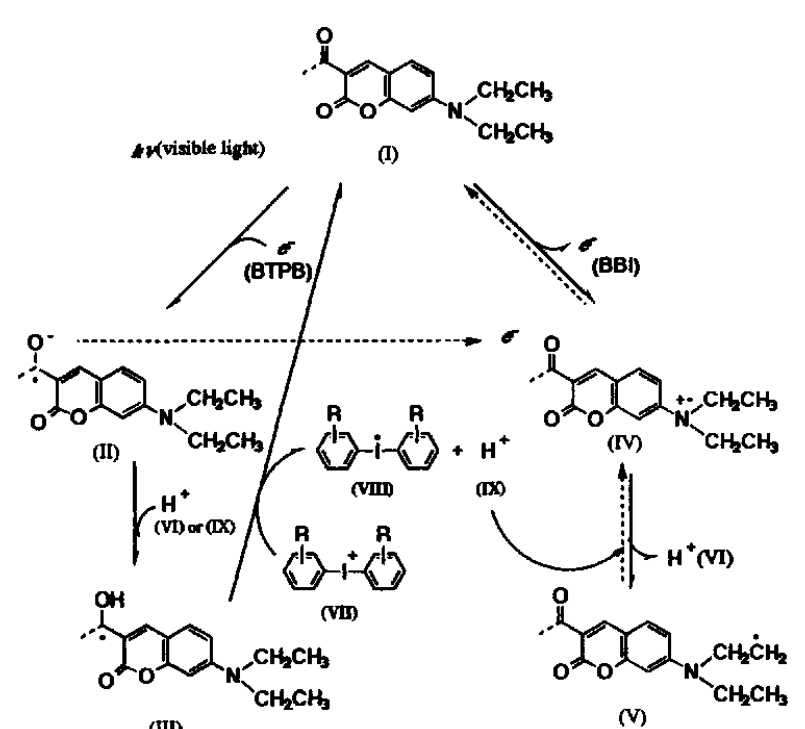

(iII)

Scheme 2. Proposed mechanism for KCD sensitized system. 
The expected reaction mechanism for the KCD, BBI and BTPB in photopolymer is depicted in Scheme 1. Thus, the KCD absorbs visible light which initially generates the excited state of $\mathrm{KCD}\left(\mathrm{KCD}^{*}\right)$. Next, $\mathrm{KCD}^{*}$ accepts an electron from BTPB, and at the same time, donates an electron to BBI. Consequently, the produced butyltriphenylboranyl radical and onium radical decompose to form the butyl radical and aryl radical. These radicals initiate polymerization of the acrylic monomer. On the other hand, the produced onium radical generates a Broonsted acid which are capable subsequently of initiating the cationic polymerization. This was confirmed by photo-DSC with 3,4-epoxycyclohexylmethyl-3',4'-epoxycyclohexane carboxylate / KCD / BBI system although this epoxide could not polymerized with KCD / BTPB system as shown in Figure 5.

Figure 6 shows spectral sensitivity curves of photopolymers consisted of $7.5 \mathrm{wt} \%$-BBI / 0.25 wt\%-KCD system (A) and $7.5 \mathrm{wt} \%$-BBI / $0.1 \mathrm{wt} \%$ KCD / 0.5 wt\%-BTPB system (B). These of both types have almost same shapes at $450-\mathrm{nm}$ or above.

Scheme 2 shows expected reaction mechanism of the KCD in photopolymer. Ketyl radicals(III) are generated by the acceptance of an electron from the BTPB and at the same time by the acceptance of a proton from the internal system such as those generated from (VI) or (IX). Since the ketyl radicals are reacted with $\mathrm{BBI}$, some of KCD are regenerated.[9] Moreover, new aryl radicals generate from reacted with BBI and the ketyl radicals. It is possible for this scheme to explain that the photosensitivity is capable of be maintained in spite of being diminished by the addition of KCD.

\section{Reference}

1. A.Umehara, J.Photopolym.Sci.Technol. 2(1989) 279.

2. Y.Ohe, Kino Zairyo 18 No.5(1998) 12.

3. T.Hirasawa, J.Photopolym.Sci.Technol.1(1988) 6.

4. K.Ichimura, A.Kameyama and K.Hayashi, $J$. Appl.Polym.Sci. 34(1987) 2747.

5. Y.Bi and D.C.Neckers, Macromolecules 62 (1996) 1877.

6. H.Ito, Y.Ohe and N.Watanabe, Proc.SPIE 2688 (1996) 2.

7. J.P.Foussier and S.K.Wu, J.Appl.Polym.Sci. 44 (1992) 1779.

8. Y.Toba, M.Yasuike and Y.Usui,Chem. Commun.(1997) 675.

9. J.P.Foussier, D.Ruhlmann, B.Graff, Y.Takimoto, M.Kawabata and M.Harada, J.Imag.Sci. Technol. 37(1993) 208. 